\title{
Image acquisition using artificial microwave radio lightning
}

\author{
Alexander $S$. Dmitriev ${ }^{1,{ }^{*}}$, Vadim V. Itskov ${ }^{1}$, Manvel M. Petrosyan ${ }^{1,2}$, Maxim G. Popov ${ }^{1}$, \\ and Anton I. Ryzhov ${ }^{1}$ \\ ${ }^{1}$ Kotelnikov Institute of Radioengineering and Electronics of Russian Academy of Sciences, 125009 \\ Moscow, Russia \\ ${ }^{2}$ Moscow Institute of Physics and Technology, 141701 Dolgoprudny, Russia
}

\begin{abstract}
The experimental analysis of image acquisition in microwave band is carried out in the article. Several radiolight lamps based on ultrawideband chaotic generators are used as multiple lightning sources. Spatial images are obtained with a help of narrow radiation pattern antenna scanning the area of interest. Compact items characterized by different electromagnetic properties were used as observation objects. It is shown that proposed method of image acquisition allows one to detect objects lit by artificial radiolight sources.
\end{abstract}

\section{Introduction}

One of the electromagnetic radiation of visible light (for example, sunlight) features is its incoherence and noise-like character, that allows our eye to receive images of the environment without interference effects that accompany coherent radiation. Incoherence is a property of thermal sources in other ranges of electromagnetic spectrum, including microwave and radiolight.

In the articles [1-3], compact simple artificial sources of radiolight were introduced based on dynamic chaos in microwave range. They allow us to light up separated areas in space, but one still needs to have equipment that is capable of receiving such signals. Investigations in this direction led to the development of radiolight cell receiver $[4,5]$. These receivers accompanied by the presence of compact radiolight sources made it possible to start investigation of radiolight fields in enclosed room [6]. This work is dedicated to the study of images acquired by illumination of rooms with several radiolight sources.

\section{Methodology and equipment}

The experimental complex includes: sources of radiolight based on dynamic chaos with a radiation range of 3.0-5.0 GHz and a radiation power of $2 \mathrm{~mW}$ each; sensitive element of radiolight receiver $[4,5]$ and parabolic antenna with horn UWB-irradiator $(2.0-7.0 \mathrm{GHz}$, the width of the radiation pattern is about $10^{\circ}$ both in the horizontal and vertical planes) [7],

* Corresponding author: chaos@cplire.ru 
together forming a radiolight receiver with a spatial resolution; personal computer (PC) with software for the radiolight receiver.

The spatial image of illuminated by radiolight area was created by scanning with antenna beam in horizontal and vertical planes, measuring the incoming signal power on the receiver depending on the angle of rotation of the antenna in the azimuth plane and in the plane of elevation angle, the scanning area was determined by the geometrical sizes of the objects of interest.

The characteristics of a parabolic antenna with horn UWB-irradiator were measured in advance using a radiolight lamp (band $3.0-5.0 \mathrm{GHz}$ ) as a radiation source. Radiation patterns of the antenna (signal power was measured) for the horizontal and vertical planes are shown in Fig. 1. The width of the radiation pattern in the horizontal and vertical planes at the $-3 \mathrm{~dB}$ level is about $10^{\circ}$.
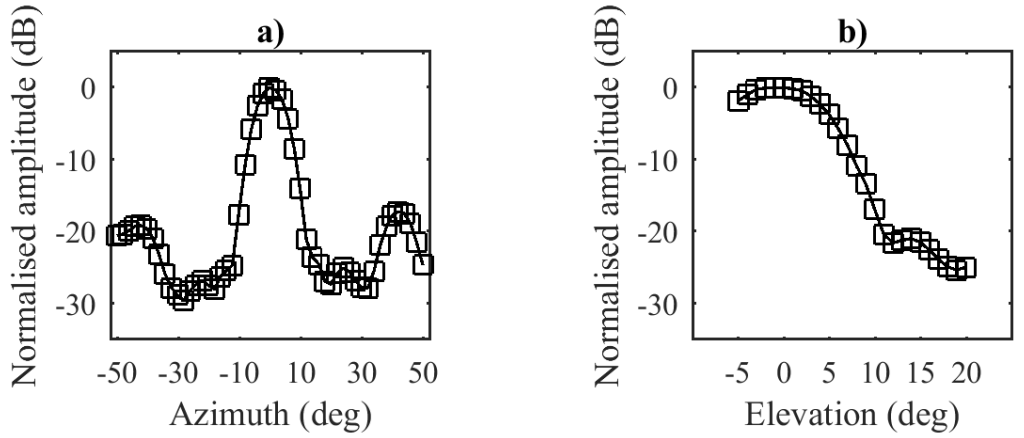

Fig. 1. Radiation power patterns for a parabolic antenna with a horn UWB-irradiator measured with a radiolight lamp (3-5 GHz band) as a radiation source: a) in the horizontal plane, b) in the vertical plane.

\section{Image acquisition using multiple radiolight sources}

Illumination of the conference room (as an example of enclosed room) in the radiolight was studied. It was revealed that the presence of a switched-on radiolight source leads to an increase in the average illumination level of the room by $25 \ldots 30 \mathrm{~dB}$ compared to the background level of illumination.

After that, a series of experiments was carried out to obtain an image of the conference room with multiple radiolight sources. First of all, it was necessary to obtain an image of the hall with switched-off radiolight lamps in order to evaluate the contribution to the illumination of uncontrolled radio sources (thermal radiation, cellular stations, Wi-Fi routers, etc.) and understand how these sources will affect subsequent experiments with radiolight lamps and what degree of spatial illumination is provided by the switched-on sources of radiolight.

The photo of the conference room and the placement of the equipment during the measurements is shown in Fig. 2: receiving device, radiolight lamps (6 pieces) placed in the standard lamp holder of the lighting chandeliers (one radiolight lamp on the chandelier) and objects for research. The receiver with a directional antenna during the majority of the experiments was placed in the part of the hall opposite to the scene. The receiving antenna was located $15 \mathrm{~m}$ apart from the far wall of the hall and $5.5 \mathrm{~m}$ apart from the closest sidewall. Radiolight sources were switched on either in groups (two and three devices), or all at the same time.

To obtain an image in the radiolight, the antenna beam was used to scan the conference room in a horizontal plane with a step of $2^{\circ}$ and in a vertical plane with a step of $2.5^{\circ}$. 
a)

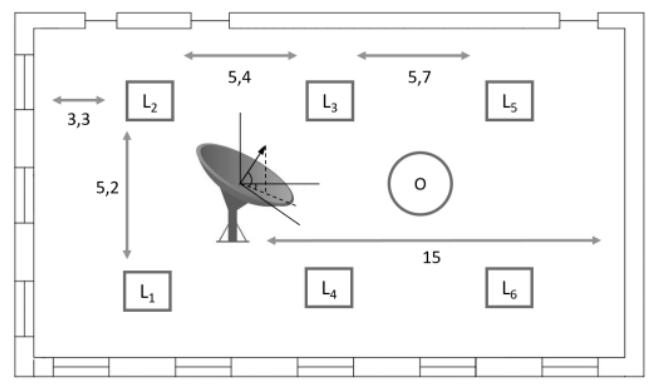

b)

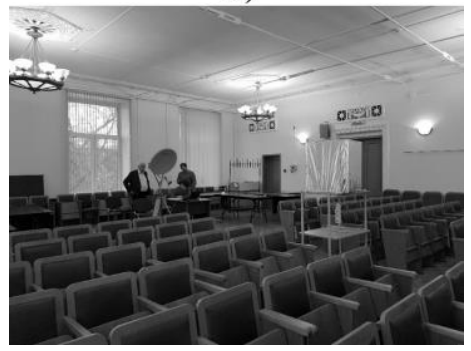

Fig. 2. Scheme of experiments (a) and photo of conference room (b); L1-L6 - radiolight lamps.

The image of the hall obtained by the described scanning process with switched off lamps of radiolight is shown in Fig. 3a. Its analysis showed that the level of the received signal ranges from -83 to $-60 \mathrm{dBm}$. In this case, there is a rather large unevenness of the background illumination depending on the direction. The greatest intensity is observed in the region of the location of the windows and in the middle of the hall: two pronounced spots are clearly visible. The spot on the right side can be caused by external sources, the radiation of which reaches the antenna through windows (Fig. 3b). The brightest element in fig. 3a corresponds to $-60 \mathrm{dBm}$ power, the darkest reaches $-83 \mathrm{dBm}$, the average value in the image is $-73 \mathrm{dBm}$.
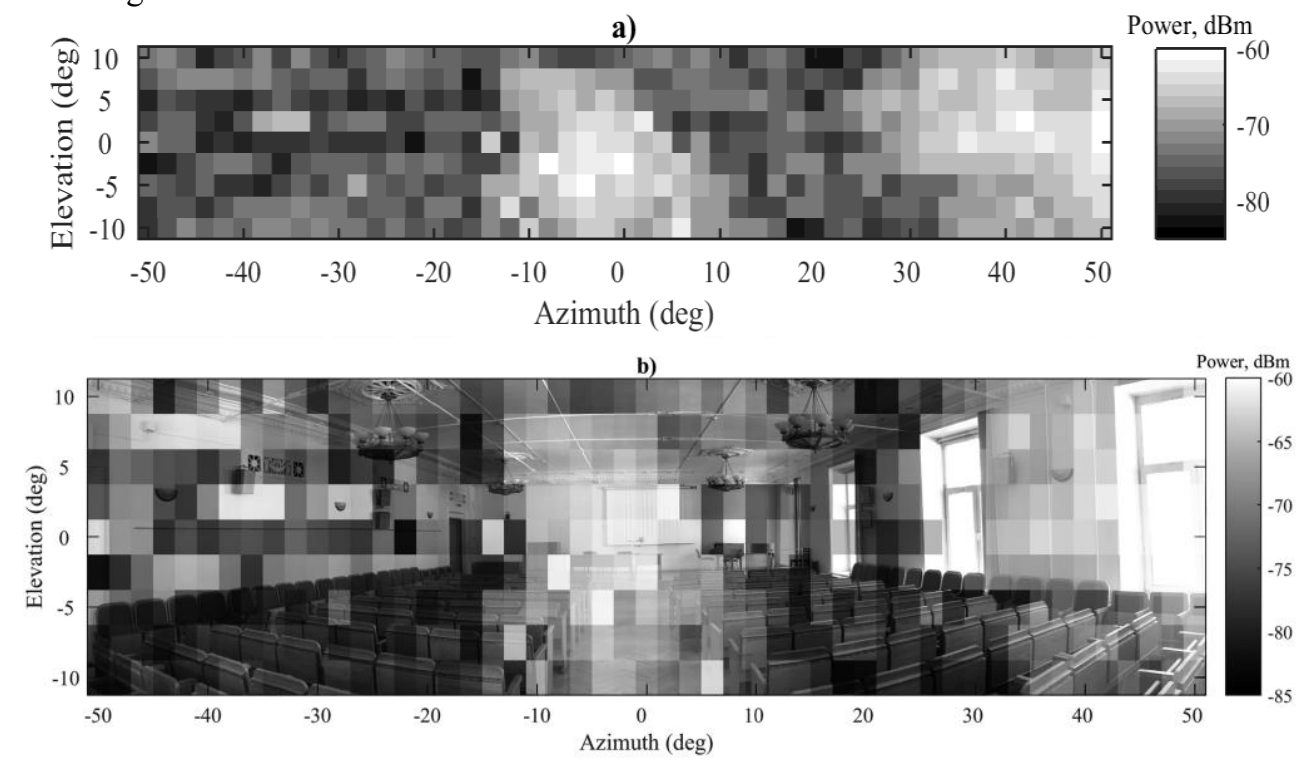

Fig. 3. The image of the hall in the microwave range without radiolight illumination (a), the composition of two images: one is in visible light and the other one is in radiolight (b).

In the next experiment the conference hall was lit with six radiolight sources located in the chandeliers above the floor at a height of about $3 \mathrm{~m}$. Fig. 4a shows a hall image obtained in radiolight for this scenario. The intensity of the received radiation was measured by directional antenna that scanned the area in the angle range of $-50^{\circ} \ldots+50^{\circ}$ with a step of $2^{\circ}$ in azimuth plane, and $-10^{\circ}$ in $+15^{\circ}$ with a step of $2.5^{\circ}$ in elevation plane.

The images of the four lamps of the radiolight in front of the antenna are clearly visible in Fig. 4a. This could be confirmed by the overlay of two images: one built in radiolight and the other one is the photographic image, shown in Fig. 4b. 

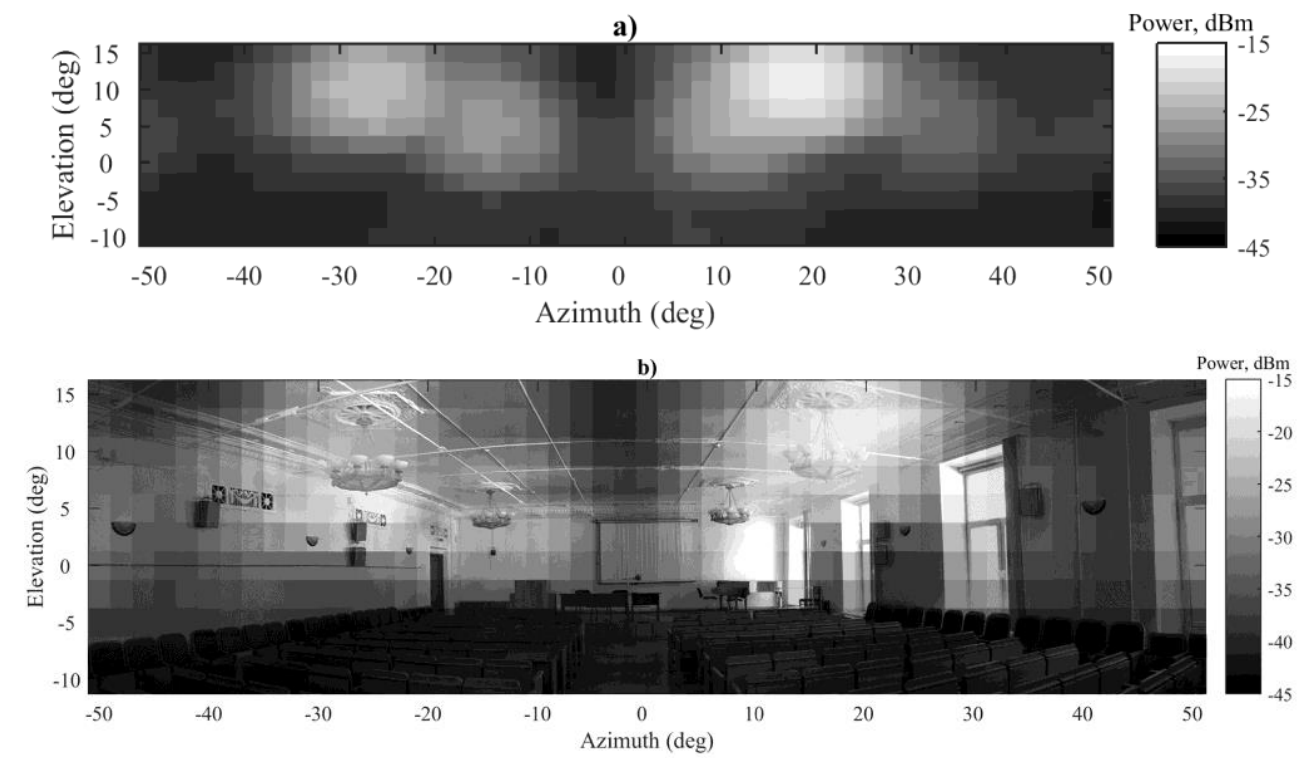

Fig. 4. Image of the hall lit by radiolight lamps (a); overlay of radiolight image with photo image (b).

Fig. 4 also shows that radiolight lamps created light "clouds" around the chandeliers. The power displayed in pixels of the figure varies from -18 to $-35 \mathrm{dBm}$ from the center to the border of the "clouds" created by the radiolight lamps. In the rest points of the image, the illumination intensity turned out to be quite uniform and varied in the range of $-40 \ldots-38 \mathrm{dBm}$.

The important point is that when the lamps are switched on, the illumination increases on average by more than $30 \mathrm{~dB}$ relative to the background level, and it is safe to say that the room is quite brightly lit by radiolight. At the same time, provided illumination of the area outside the chandeliers in the hall is uniform and sufficient. This illumination allows us to proceed to experiments that study the characteristics of radio images of objects with different electromagnetic properties.

\section{Compact objects image}

During the selection of objects for study, attention was paid to their size (it should have been at least half a meter) and electromagnetic properties. In accordance with the second criterion, the following objects were selected: a cube covered with metal foil (two versions see below); a cuvette filled with water (dielectric constant 81, refractive index 9) sheets of radio absorbing material.

\subsection{First group of experiments}

The first experiments were carried out to find out how the illumination of the area changes when a metal cube appears. The image was obtained in the range of angles $-10^{\circ} \ldots+10^{\circ}$ with steps of $2^{\circ}$ in azimuth plane, and angles $-10^{\circ} \ldots+5^{\circ}$ with steps of $2.5^{\circ}$ in elevation plane.

The cube was located at a distance $d$ in front of the antenna, the front face of the cube was exposed perpendicular to the direction of the antenna. Experiments were performed for distances d equals to 2, 4, and $5 \mathrm{~m}$. In Fig. 5, the images were obtained in the experiment when the distance between the receiver and the cube was $5 \mathrm{~m}$ : the background image with six lamps switched on and no cube (Fig. 5a) and with the lamps switched on in the presence 
of a cube (Fig. 5b). When comparing Fig. 5a and 5b, there is some difference, but it is difficult to identify the appearance of a new item in the image. However, its presence is clearly traced in Fig. 5c, obtained by constructing a differential image by subtracting the image in Fig. 5a from the image in Fig. 5b. In this case, the dynamic range of the differential image (contrast) is about $2 \mathrm{dBm}$. The cube area attenuates the signal compared to the background image.
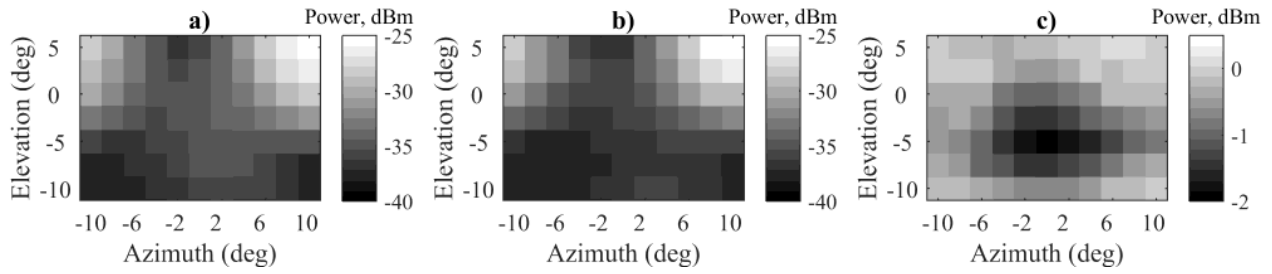

Fig. 5. Obtaining an image of a cube: a) background image with switched on radiolight lamps and the absence of an object; b) the image of a cube located in front of the antenna at a distance of $5 \mathrm{~m} ; \mathrm{c}$ ) differential image.

In the upper corners of Fig. 5 bright areas corresponding to the sources of radiolight are clearly visible. Their presence "light up" the rest of the image and interferes with their visualization. In order to avoid this negative effect, in the subsequent experiments four lamps of the radio light of the front part of the conference room were switched off (lamps L3-L6 in Fig. 2a).

As noted above, the radiation intensity in the presence of a cube is lower than in its absence. This may be caused by the reflection of direct rays falling on cube's front face from two radiolight sources so that their further propagation differs from the direction to the receiver antenna.

If this assumption is correct, then the cube will be the source of an amplified signal at the receiver input in two cases: 1) the cube face is a "rough" surface, diffusely reflecting the rays falling on it; 2) the cube is positioned so that incidence angle of radiation from the radio source to the surface of the cube is equal to the angle between the direction of maximum of the antenna radiation pattern and the normal to the surface of the cube. To check these scenarios, the corresponding experiments were carried out with amplifying objects (objects that amplify signal at the input of radiolight receiver).

Irregular roughness with characteristic dimensions of the average wavelength of the radiolight was created on the surface of the cube (Fig. 6a) to conduct first series of experiments.

a)

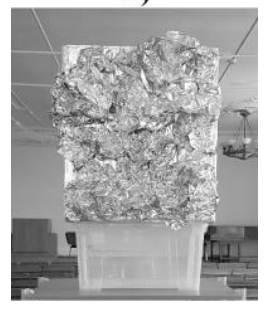

b)

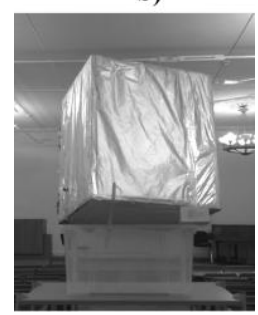

Fig. 6. Experiments with amplifying objects: a) a cube with a rough surface; b) a cube with a mirror surface that reflects the beam from the front face in the direction of the antenna.

The results of experiments with a cube having a rough surface are shown in Fig. 7. The image is built in the range of angles $-14^{\circ} \ldots+14^{\circ}$ with a step of $2^{\circ}$ in azimuth plane, and $10^{\circ} \ldots+10^{\circ}$ with a step of $2^{\circ}$ in elevation plane. 
Experiments have shown that the addition of scattered radiation from the surface of the cube was sufficient to detect the presence of the cube in Fig. $7 \mathrm{~b}$ relative to the background in Fig. 7a even without additional processing. In the image shown in Fig. 7c, the appearance of the cube increased the signal at the receiver output relative to the background by $2 \ldots 5 \mathrm{~dB}$. The bright spot on the top of Fig. $7 \mathrm{c}$ is explained by the fact that switching off four lamps (see lamps L3-L6 on Fig. 2a) to eliminate the excessive exposure of the receiver to direct rays from the lamps contributed to reduction of uniformity of illumination in the far area of the hall.
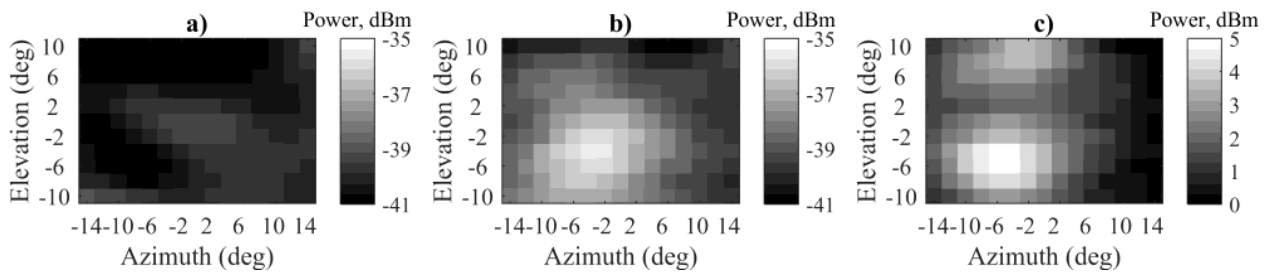

Fig. 7. Cube with a rough surface: a) background image from radiolight lamps; b) cube is located in front of the antenna at a distance of $2 \mathrm{~m}$; c) differential image.

During the second experiment, the cube was located so that the beam reflected from one of the two radiolight lamps (see Fig. 6b) fell into the receiving antenna. The distance between the cube to the antenna was $2 \mathrm{~m}$.

The images in Fig. 8 were obtained in the range of angles from $-24^{\circ}$ to $+24^{\circ}$ with steps of $2^{\circ}$ in azimuth plane, and from $-10^{\circ}$ to $+20^{\circ}$ with steps of $2.5^{\circ}$ in elevation plane.
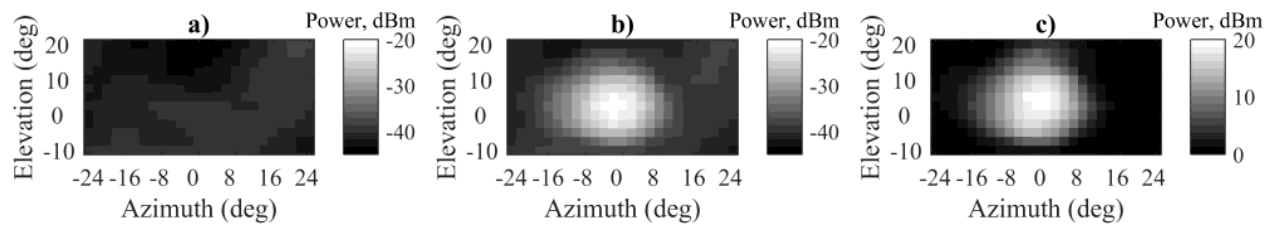

Fig. 8. Image of cube, the beam reflected from its surface falls directly into the receiving antenna: a) background image from radiolight lamps; $b$ ) the image of the cube in the radiolight $c$ ) differential image.

As in the case of the cube with a rough surface, the reflected beam in this scenario gave a sufficient additional power at the receiver input to detect the object without the construction of differential image (Fig. 8b). The reflected radiation increased the signal level at the receiver output by $5 \ldots 20 \mathrm{~dB}$ (Fig. 8c). The increase in the received signal power in this case significantly exceeded the one from the scattered radiation in the previous experiment. In addition, one should note that a high level of reflected radiation from the cube leads to a decrease of the influence of illumination irregularity level on the differential image (see Fig. 8c).

\subsection{Second group of experiments}

To clarify the possibility of observing objects from a dielectric and absorbing materials in a radiolight, a cuvette filled with water and sheets of radio absorbing material were used as objects of interest.

A cuvette filled with water was placed $2 \mathrm{~m}$ apart from the receiving antenna. The object of study was not reflective and had a high dielectric constant $\varepsilon=81$. The antenna was additionally tilted forward so that the angular dimensions of the cuvette were from $-10^{\circ}$ to $+8^{\circ}$ in the azimuth plane and from $0^{\circ}$ to $-10^{\circ}$ in the elevation plane. 
The level of received radiation from the cuvette direction was lower than in its absence. The dynamic range of the differential signal (contrast) was about $3 \mathrm{~dB}$ (Fig. 9). The presence of a cuvette filled with water could be observed only on the differential image.

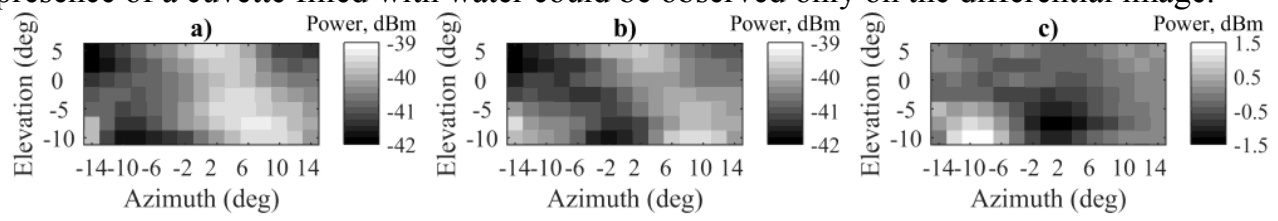

Fig. 9. Images of a cuvette filled with water in a radiolight: a) a background image (there is no cuvette), b) an image with a cuvette, c) a differential image.

Next, an experiment was conducted using three square sheets of absorbing coating positioned $2 \mathrm{~m}$ apart from the receiving antenna along the horizontal line. The angular dimensions of the object were: the azimuth angle ranged from $-20^{\circ}$ to $+20^{\circ}$, and the elevation angle ranged from $-6^{\circ}$ to $+5^{\circ}$.

As in the case of the cuvette filled with water, the level of received radiation from the object direction was lower than in its absence. In this scenario, the contrast of the obtained image turned out to be comparable with the contrast of the differential image of the cuvette filled with water, about $3 \mathrm{~dB}$.

\subsection{Third group of experiments}

In order to find out the possibility of observing various objects in the radiolight behind the obstacles (for example, the walls of buildings), experiments were conducted to create images of objects located behind the wall relative to the receiver.

In the experiments, the receiving antenna was located in the conference room $1 \mathrm{~m}$ apart from the wall adjacent to the corridor, and the observed object $(\mathrm{O})$ was located on the opposite side of the wall, in the corridor, $2 \mathrm{~m}$ apart from the wall and there was also a radiolight lamp (L) placed at the floor level. The object was positioned at a height of about $1.5 \mathrm{~m}$ above the floor. This was done in order to illuminate the object of interest and not to illuminate the receiving antenna with a direct beam from the radiolight lamp.

In $[4,5]$, it was found that there is an additional signal attenuation of $6 \ldots 8 \mathrm{~dB}$ when a chaotic UWB signal propagates through such wall.

A cube with a diffusely reflecting surface mentioned above was chosen as the object of the experiment placed behind the wall, for which in previous experiments a high degree of image contrast relative to the background illumination level was obtained.

All images created during the experiments were built in the azimuth angles ranged from $-30^{\circ}$ to $+30^{\circ}$ and elevation angles $-10^{\circ}$ to $+10^{\circ}$.

First, an image of the background radiation in the corridor (the location of the object) was obtained with the switched-on radiolight lamp (Fig. 10a).

The background radiation level here was about $-53 \ldots-60 \mathrm{dBm}$, while a similar measurement of the background radiation in the conference room was $-38 \ldots-40 \mathrm{dBm}$.

Next, a cube image was obtained (Fig. 10b). This image clearly shows the borders of the cube. The dynamic brightness range of pixels per image is $\sim 15 \mathrm{~dB}$. Comparing this picture to the image in Fig. 10a, one can see the gain of the received signal in the area where the cube is located is $10-12 \mathrm{~dB}$. For clarity there is Fig. 10c presenting differential image of the received signal.

Comparing the obtained images of the cube behind the wall and the cube in the conference room, one can realize that the contrasts of the images between the cube area and the area adjacent to it in both images are about the same level of $6 \ldots 8 \mathrm{~dB}$, despite the 10 $\mathrm{dB}$ difference in the average illumination level of scenes in the conference room and with the presence of a wall in a line of sight between the object and receiver. 

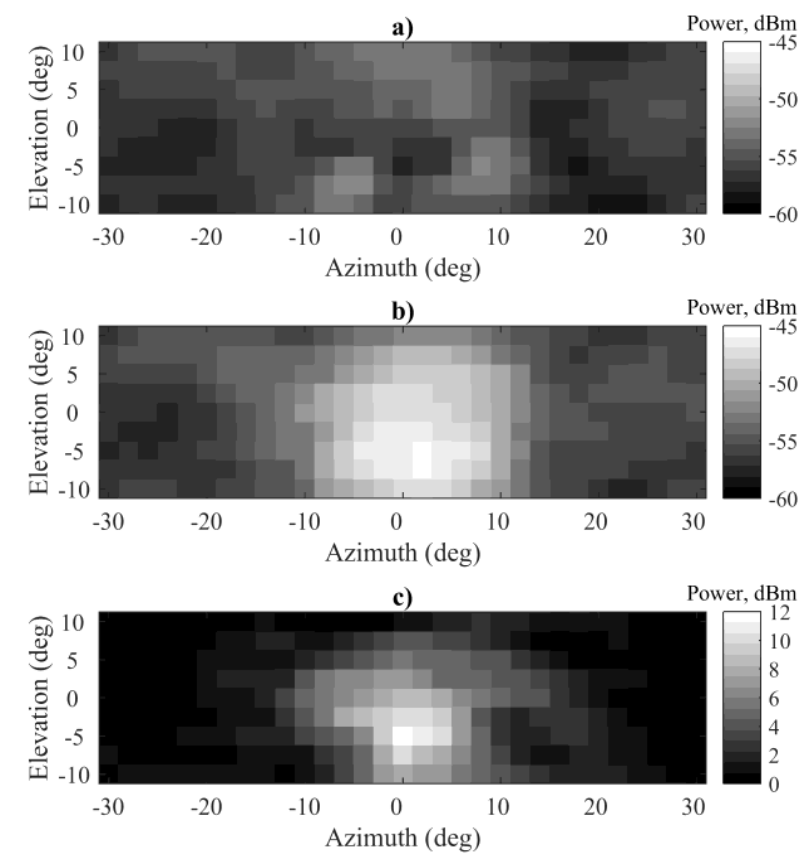

Fig. 10. Background image of the corridor in the radiolight (a), the image of the cube behind the wall in the radiolight (b), the differential image (c).

\section{Conclusion}

Image acquisition method of the environment area using incoherent microwave radiation from radiolight lamp as a source of illumination of the area and radiolight receiver based on sensitive element and directional antenna is proposed and tested. The conducted experiments made it possible to acquire images of the area of interest in microwave band as well as to detect the presence of new objects in the observable area.

The work was carried out within the framework of the state task.

\section{References}

1. A.S. Dmitriev, E.V. Efremova, M.Yu Gerasimov, V.V. Itskov, J COMMUN TECHNOL EL+, V 61, no 11, 1073-1083 (2016)

2. A.S. Dmitriev, E.V. Efremova, Technical Physics Lett., V 42, no 24, $49-57$ (2016)

3. A.S. Dmitriev, E.V. Efremova, Nonlinear Phenomena in Complex Systems, V 20, no 2, 133 (2017)

4. Yu.V. Gulyaev, A.S. Dmitriev, V.A. Lazarev, T.I. Mokhseni, M.G. Popov, J COMMUN TECHNOL EL+, V 61, no 8, 894-900 (2016)

5. Yu.V. Gulyaev, A.S. Dmitriev, V.V. Itzkov, M.M. Petrosyan, A.I. Ryzhov, A.V. Uvarov, J COMMUN TECHNOL EL+, V 63, no 9, 1-7 (in Russian) (2018)

6. A.S. Dmitriev, V.V. Itskov, M.M. Petrosyan, M.G. Popov, A.I. Ryzhov, Physical Bases of Instrumentation, V 7, no 3(29), 50-63 (2018)

7. V.I. Kalinichev, V.A. Kaloshin, L.I. Pangonis, Trudy III Vseros. konf. «Radiolokaciya $i$ radiosvyaz'», 101 (in Russian) (2009) 